\title{
In vivo and in vitro techniques for comparative study of antiviral T-cell responses in the amphibian Xenopus
}

\author{
Heidi Morales ${ }^{1}$ and Jacques Robert ${ }^{1 \#}$
}

\author{
${ }^{1}$ Department of Microbiology and Immunology, University of Rochester Medical Center, Rochester, NY 14642, USA \\ \#Corresponding Author: Dr. Jacques Robert, Ph.D., Department of Microbiology and Immunology, University of Rochester Medical \\ Center, Rochester, NY 14642, USA. Phone: (585) 275 1722; Fax: 716473 9573; E-mail: jacques robert@urmc.rochester.edu
}

Submitted: September 6, 2007; Revised: September 24, 2007; Accepted: September 26, 2007

Indexing terms: Xenopus laevis; ranavirus; T-Lymphocytes

Abbreviations: Ag, antigen; APBS, amphibian phosphate buffered saline; BrdU, bromodeoxyuridine; CFSE, carboxyfluorescein diacetate succinimidyl ester; FACS, fluorescence activated cell sorting; mAb, monoclonal antibody; MHC, major histocompatibility complex

\section{ABSTRACT}

Activation of lymphocytes in mammals is often quantified by measuring the amount of proliferation during the expansion phase of an immune response. Bromodeoxyuridine (BrdU) incorporation and carboxyfluorescein diacetate succinimidyl ester (CFSE) dilution assays are some of the techniques widely used in mammalian studies of pathogen-induced proliferation and provide a convenient way of quantifying the cellular response. We have extended the use of these proliferation assays to the amphibian Xenopus laevis. We have developed this species as a valuable comparative model to study immunity against a well-known amphibian pathogen, Frog Virus 3 (FV3). Fluorescence activated cell sorting was used to assess the level of BrdU incorporation of lymphocytes in vivo and CFSE dilution in an in vitro activation assay. Both techniques have shown that splenic lymphocytes proliferate specifically upon FV3 challenge. This indicates that common methods for detection of proliferation upon immunologic challenge are easily applied to other vertebrate species, as it highlights the evolutionary conservation of the proliferative nature of immune responses throughout vertebrate phyla.

\section{INTRODUCTION}

At the start of an immune response in lymphoid organs, naive $\mathrm{T}$ lymphocytes are activated and proliferate in response to antigens (Ags) presented in the context of the major histocompatibility complex (MHC) receptors by professional antigen presenting cells (APCs). Effective pathogen clearance depends on the clonal expansion and maturation of $\mathrm{T}$ lymphocytes into $\mathrm{T}$ cell effectors that can recognize and act against specific Ags (1). Therefore, the measure and quantification of the level of proliferation in vivo in lymphoid organs as well as in vitro in cell cultures has become a staple of immune response assessment. The thymidine incorporation assay is a very common technique that measures lymphocyte responses by determining the amount of radioactive nucleotide incorporated into the DNA of proliferating cells in an in vitro culture after exposure to activating stimuli (2,3). A higher cell number due to proliferating cells results in a higher radioactive signal. Methods that use fluorescence activated cell sorting (FACS) to identify populations of proliferating cells have proven convenient and safe since they require no radioactivity and are more informative since they allow monitoring proliferation of distinctive cell populations identified by specific markers on their cell surface $(4,5)$. Proliferation assays using multiparametric FACS have allowed the identification of differentially expanding $\mathrm{T}$ cells both in vivo (6) and in vitro (7) and have revealed the kinetics of $\mathrm{T}$ cell activation upon antigenic challenge over time. The two main methods widely used in mammals are bromdeoxyuridine (BrdU) incorporation and carboxyfluorescein diacetate succinimidyl ester (CFSE) staining.

BrdU is a thymidine analogue that incorporates into newly synthesized DNA of cells at the S phase of the cell cycle (8). Antibodies specific to BrdU are used to determine the presence 
of the molecule in the genomic DNA and the number of cells that have incorporated it. Because BrdU is intracellular, antibodies specific to surface molecules can also be used to identify the cell types incorporating $\mathrm{BrdU}$ in a given population. The use of a specific antibody to nascent DNA makes it a useful technique for immunohistochemical, cytometric and microscopic analyses (9).

An alternative to BrdU incorporation assays, and not based on DNA synthesis, is the use of CFSE, a membrane permeable non-toxic fluorescent dye also widely used for the detection of immune cell proliferation in vitro (10). CFSE incorporates at similar levels into all cells but dilutes two fold at each cell division. This permits the detection of up to 10 cellular divisions and therefore provides a more robust quantification of the proliferation by a cell population than BrdU incorporation (5).

Whereas activation and expansion of $\mathrm{T}$ cells during antiviral responses are well characterized in mammals (11-14) very little is known about the proliferative capacity of activated $\mathrm{T}$ lymphocytes against viral pathogens in cold-blooded vertebrates. In one account, cells isolated from trout kidneys were shown to proliferate in response to adherent cells that had been stimulated previously with a recombinant viral protein that act as antigen presenting cells (15). However, due to the lack of antibodies that recognize specific surface markers in this species, a more detailed analysis of the nature of proliferating cells is missing. We have established Xenopus as an important model with which to study immunity against ranaviruses (Iridoviridae) in amphibians (16) and showed evidence of the critical role of splenic lymphocytes, in particular CD8 T cells, in host resistance to Frog Virus 3 (FV3) infection (17). Proliferation of splenic CD8 T cells upon FV3 infection has been recently characterized in vivo using a BrdU incorporation method and fluorescence activated cell sorting (FACS) (18). In this review, we describe in detail these methods, adapted for Xenopus, to monitor the proliferating responses to virus of splenocytes, including total $\mathrm{T}$ cells, CD8 $\mathrm{T}$ cells and $\mathrm{IgM}+\mathrm{B}$ cells.

\section{MATERIALS AND METHODS}

\section{Animals, reagents and FV3 stocks}

Two-year old (about 3 in. long) outbred Xenopus adults, and monoclonal antibodies (mAbs) specific to Xenopus CD8 (AM22; 19), Class II (AM20, 19) and IgM (10A9; 20) were obtained from the $X$. laevis Research Resource for Immunobiology at the University of Rochester Medical Center (http://www.urmc.rochester.edu/smd/mbi/xenopus). Frog Virus 3 (FV3, Iridoviridae) was grown in and purified from A6 Xenopus kidney cell line as previously described (16). Viral titers were determined using A6 cells by the $50 \%$ endpoint dilution method. Randomized groups of frogs were inoculated by intraperitoneal injection of $5 \times 10^{6}$ pfu of FV3 in $300 \mu$ of PBS modified to amphibian osmolarity (APBS). All animals were handled under strict laboratory and UCAR regulations (Approval number 2004-199), minimizing discomfort at all times.

\section{In vivo BrdU incorporation assay}

Outbred adult frogs were injected once with $3 \times 10^{6}$ pfus FV3 and incubated for 3, 6, 9 and 14 days. Frogs were then incubated in $100 \mathrm{ml}$ of water containing $1 \mathrm{mg} / \mathrm{ml}$ bromodeoxyuridine (BrdU, Sigma St. Louis, MO; Catalog \#B5002-1G) 2 days before sacrifice. Splenocytes were washed 2x in APBS, counted and stained for surface markers CD8 (AM22, IgM isotype), Class II (AM20; IgG1 isotype) and IgM (10A9; IgG1 isotype) followed by APC conjugated Goat anti Mouse mAb (Accurate JGM136146). Cells were washed in APBS/0.05\% Tween to permeabilize and treated with DNase (Sigma DN25-1G) followed by incubation with FITC conjugated anti BrdU mAb (Roche 1202693). Cells were then washed and analyzed by flow cytometry using a FACS Calibur (BD Biosciences). 20,000 events were collected and the lymphocyte population was gated based on their forward and side scatter profiles.

\section{In vitro CFSE proliferation assay}

Peritoneal leukocytes (PLs) from outbred frogs infected once for 3-weeks with $3 \times 10^{6}$ pfu FV3 (primed) or uninfected (unprimed) were isolated by peritoneal lavage (2), washed with APBS and infected with FV3 at 1 multiplicity of infection (MOI) for 24 hours in $1 \mathrm{ml}$ amphibian grade culture medium (ASF-A6) in 24 well plates. Twenty-four hours later, spleens harvested from the same outbred frogs were scraped using a nylon mesh and washed two times with APBS. Cells were labeled with $5 \mu \mathrm{M}$ CFSE (Sigma 21880) for $20 \mathrm{~min}$. at $27^{\circ} \mathrm{C}$ in the dark and washed 3 times with APBS. Splenocytes were added to infected PLs at a $1: 1$ ratio for 24,48 and 72 hours. For FACS analysis, total coculture cells were washed twice in APBS and stained using Xenopus specific mAbs, as mentioned above. 


\section{RESULTS AND DISCUSSION}

Anti-viral lymphocyte proliferative responses monitored in vivo by BrdU incorporation in Xenopus

To assess the in vivo proliferative response of Xenopus splenocytes during viral infection, we adapted the BrdU incorporation assay used in the mouse model (5). Adult frogs were injected intraperitoneally with the iridovirus FV3 and treated with $1 \mathrm{mg} / \mathrm{ml}$ of BrdU directly added in the water 2 days before sacrifice. A representative experiment is depicted in Fig. 1A. Forward scatter and side scatter profiles of Xenopus splenocytes are comparable to that of mammals, indicative of small cells with little to no granularity. The slight increase in the size of the lymphocytes (indicated by an arrow in Fig. 1 forward scatter and side scatter plots) in infected frogs compared to the uninfected controls suggests activation and consequent blasting of lymphocytes in the spleen as observed in mammals and ectothermic vertebrates (21). Similarly, the total number of splenocytes, as well as BrdU+ cells, obtained from infected frogs was substantially higher than uninfected frogs, indicating an expansion of cells due to immune activation (Fig. 1).

Gating was done on cells with size characteristics of lymphocytes (also the most prominent population in the spleen) in both infected and uninfected samples for analysis of surface markers and BrdU (Figs. 1 and 2). Although some BrdU incorporation was detected in lymphocytes from uninfected animals, a significantly higher fraction of lymphocytes in the infected frog had incorporated BrdU (Fig. 1 histograms). About $7 \%$ of total splenocytes in the infected frog incorporate BrdU versus $3 \%$ in the uninfected control. The low level of proliferation detected in uninfected animals is possibly due to the basal homeostatic proliferation of various lymphocyte subsets or an unidentified immune response in that animal, given that animals in our facilities are not germ free. To determine the cell types involved in the proliferative response upon FV3 infection, we took advantage of Xenopus specific mAbs recognizing cell surface markers such as CD8 for T cells, MHC class II for all lymphocytes and IgM for B cells. As previously described in detail (18), CD8 T cells constitute the main lymphocyte effectors proliferating in response to FV3 infection. We recently found that IgM+ B cells also undergo a marked proliferative response against FV3, albeit lower than CD8+ lymphocytes (Fig. 2, far right panels). In addition, surface IgM expression in BrdU negative cells of the infected sample was increased from $2 \%$ to $11 \%$, (Fig 2). This result further substantiates earlier observations of B cell activation during a

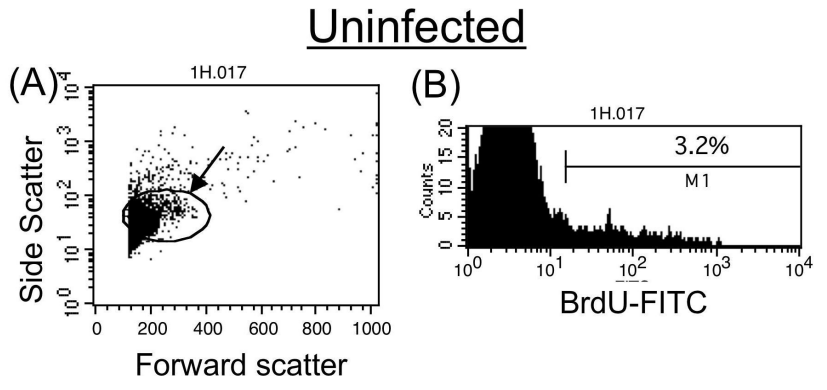

Infected

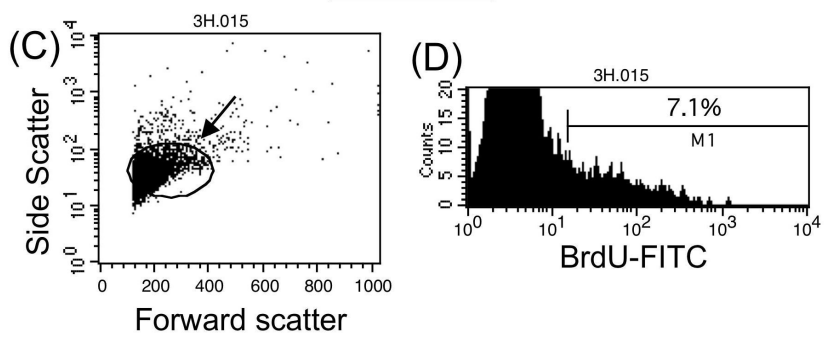

Fig. 1: Detection of Xenopus splenocyte proliferation 6 days post-FV3 infection by BrdU assay. Uninfected control frogs and frogs infected for 6 days with FV3 were treated with BrdU 48 hrs before the assay. Cells were then permeabilized, treated with DNase, stained with FITC-conjugated anti-BrdU $\mathrm{mAb}$, and analyzed by FACS (Representative of six different experiments). Ten thousand total events were gated based on side and forward scatter profiles (A, C). The arrows depict cells with higher forward scatter that increase in number in the infected sample. The percent of gated BrdU positive cells is shown in the histograms $(B, D)$.

primary FV3 response, which is necessary for the establishment of memory B cell responses observed during a secondary response (22). Furthermore, proliferation in the spleen is due entirely to immune cells since no MHC class II negative cells proliferate (e.g., incorporate BrdU) upon FV3 challenge during the times tested (Fig. 2, lower right quadrants of MHC-II dot plots).

These results show that proliferation of immune cells in the spleen of an ectothermic vertebrate upon pathogenic challenge can be monitored in vivo by a BrdU incorporation assay as it is done in mammals, and further highlights the improvement of immunologic techniques in $X$. laevis.

Anti-viral lymphocyte proliferative responses monitored in vitro by CFSE dilution assay in Xenopus

Our previous study has provided in vivo evidence of an accelerated $\mathrm{CD} 8 \mathrm{~T}$ cell response during secondary viral challenge (18). These results suggest that CD8 $\mathrm{T}$ cells were efficiently primed during the primary anti-viral response and that memory $\mathrm{T}$ cells were generated. To further characterize the Robert et al. - In vivo and in vitro techniques for comparative study of antiviral T-cell responses in the amphibian Xenopus www.biologicalprocedures.com 


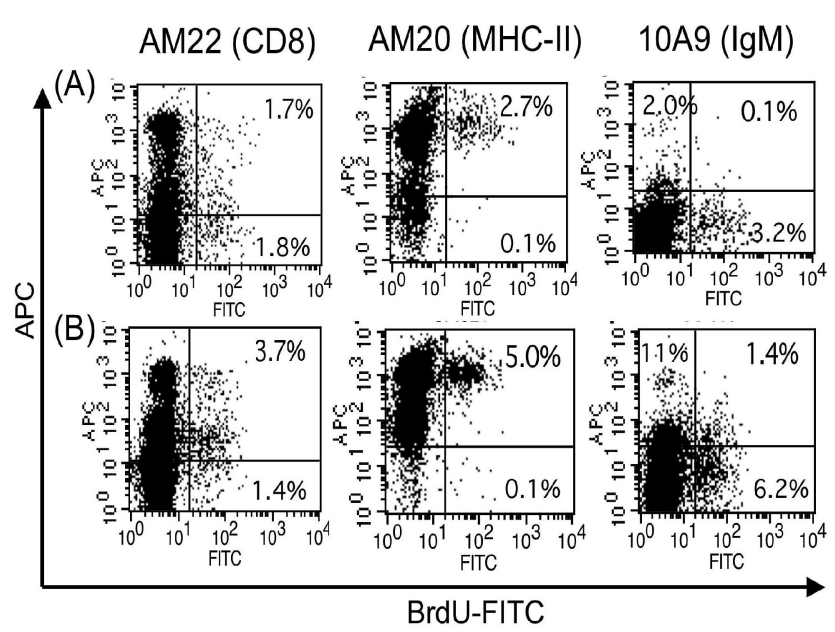

Fig. 2: Proliferation of CD8 $+T$ and IgM+ B cells 6 days post-FV3 infection detected by BrdU assay. Representative two-color flow cytometry analysis of splenocytes from uninfected control frogs (A), and frogs infected for 6 days (B). Splenocytes were surface stained for Xenopus MHC Class II (AM20), CD8 (AM22) or IgM (10A9) followed by APC-conjugated goat anti-mouse secondary antibody. Cells were then permeabilized, treated with DNase and stained with FITCconjugated anti-BrdU mAb and analyzed by FACS. Twenty thousand total events were gated based on side and forward scatter profiles as in Fig. 1. The number in the upper right quadrant indicates the percent of double positive cells.

proliferative response of primed $\mathrm{T}$ cells, we have developed an in vitro proliferation method using CFSE, which is a fluorescent dye that incorporates into all viable cells. Upon cell division, CFSE is equally distributed in each daughter cell, making it possible to measure each dividing cell from the parent cell as diluted CFSE in a population.

We have characterized peritoneal leukocytes (PLs) likely to be antigen-presenting cells in Xenopus laevis (Morales and Robert unpublished results). During FV3 infection, in vivo, cells in the peritoneum increase in number and size, and macrophage-like cells constitute roughly $30-50 \%$ of total peritoneal leukocytes. To test whether macrophage-like cells in the peritoneum act as antigen presenting cells, PLs were isolated from 3 week primed animals and infected in vitro with 1MOI FV3 for 24hours, then co-cultured with CFSE-stained splenocytes from the same animal for 72 hours. Unprimed CFSE stained splenocytes cocultured with infected PLs from the same animal were used as a negative control. The profiles of both in vitro co-cultures at time 0 shows an expected high CFSE content in splenocytes, which have not yet begun to proliferate (representative show in Fig. 3 top panel). Given the low number of PL's in the culture, no CFSE negative population is visible. The dot plots in Fig. 3A show differences in side scatter and forward scatter profiles Robert et al. - In vivo and in vitro techniques for comparative study of antiviral T-cell responses in the amphibian Xenopus www.biologicalprocedures.com

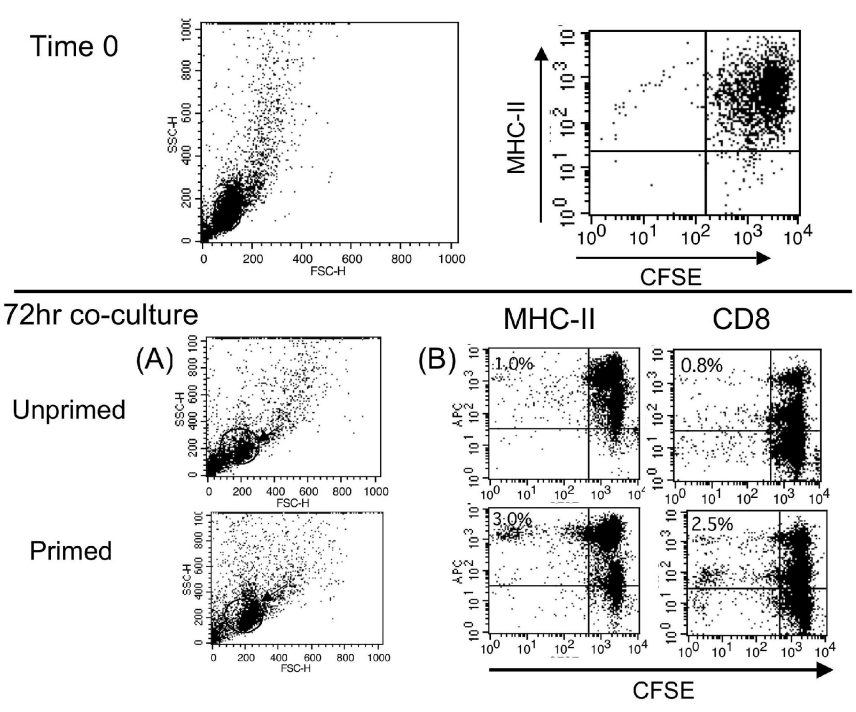

Fig. 3: Detection of Xenopus primed splenocyte proliferation induced in vitro by FV3 infected FV3 peritoneal leukocytes using the CFSE assay. Splenocytes from an uninfected control frog or a frog primed 3 weeks before the assay by infection with FV3 were CFSE stained and cocultured for 72 hours with FV3 infected peritoneal leukocytes obtained from the same frogs 3 days before the assay. Total culture was surface stained for MHC class II (AM20) or CD8 (AM22) and analyzed by FACS. A time zero analysis shows initial CFSE staining of splenocytes prior to co-culture. Analysis at 72 hrs (B) was done on gated population in the side scatter dot plot (A). Numbers in upper left quadrant indicates the percent of CD8 or Class II positive cells with diluted CFSE.

between unprimed and primed cells cultured with infected PL's for $72 \mathrm{hrs}$. Similar to what is observed in vivo with the BrdU incorporation assay, cells are slightly larger (see arrow) and more abundant within the gate in the primed samples (about $50 \%$ of total primed splenocytes versus $41 \%$ of unprimed splenocytes lie within the gate; data not shown). As shown in panel B a significant amount of proliferation, indicated by the dilution of CFSE staining, is observed (bottom panel B). This includes about a $2 \%$ increase in CFSE diluting MHC-II and CD8 positive cells. Furthermore, among the 3\% of primed MHC-II cells that display dilution of CFSE, $2 \%$ are CFSE low or negative, and most of the $2.5 \% \mathrm{CD} 8+/ \mathrm{CFSE}^{\text {dil }} \mathrm{T}$ cells are also CFSE low or negative. This is indicative of an extensive daughter cell generation. No significant increase of division is observed in co-culture between unprimed CFSE+ splenocytes and infected PLs.

The significance of these results is three fold: (i) in Xenopus both MHC-II+ cells and CD8+ T cells proliferate in vitro in response to stimulation with FV3 infected PLs; (ii) PLs can act as antigen presenting cells in vitro; and (iii) the usefulness and efficiency of this method is remarkable as it can be used in 
combination with multiple conditions to test antigen can also contribute to a considerable loss of cells. We found that presentation and pathogen specificity of which little is known in suction of the washing and staining buffers with a Pasteur ectothermic vertebrates.

To our knowledge, this is the first account of a measurable proliferative response of lymphocytes against a viral pathogen in an ectothermic vertebrate. We have shown that commonly used techniques to determine lymphocyte proliferation in mammals can also be applied to assess cellular immune responses in amphibians. We have previously shown that CD8 $\mathrm{T}$ cells involved in anti-ranaviral response in Xenopus proliferate (e.g., incorporate $\mathrm{BrdU})$ at detectable levels in the spleen, in both primary and secondary infections. Furthermore, $\mathrm{T}$ cell priming resulting from FV3 infection can be determined in vitro by coculture of primed splenocytes with antigen presenting cells infected with FV3. The use of CFSE in this instance allows a rigorous analysis of the level of proliferation of various Xenopus lymphocyte populations. It is noteworthy that in both BrdU and CFSE based assays, proliferation of activated lymphocytes was minimal compared to that observed in mammals $(4,7)$. We have suggested that the relatively small response of Хenopus lymphocytes may be due to the lack of secondary lymphoid organs such as the lymph nodes, which facilitate intimate contact with the antigen presenting cells $(22,23)$. However, since we also observe this effect in vitro, it may be due to intrinsic characteristics of the cellular potential in this ectothermic vertebrate. We speculate, for example, that the effect of cytokine secretion in Xenopus may be limited by either a relatively lower amount of secreted cytokine, diminished receptor expression in the cell surface or a lack of those cytokines specifically involved in boosting $\mathrm{T}$ cell proliferation in mammals (i.e. IL-2, IL-17 and IL-15).

Some technical issues concerning the use of BrdU and CFSE in Xenopus splenocytes are discussed below. The DNAse treatment in the BrdU incorporation assay, which opens the genomic DNA and allows the antibody access to the BrdU epitope, is damaging and results in loss of cells; about 100,000 to 500,000 (or 20-50\% of starting cell numbers) cells can be lost in a single DNase treatment. While a lower DNase concentration diminishes cell loss, it also decreases the intensity of the specific signal, and ultimately, the number of positive cells. Optimal results were obtained when the initial total number of cells per sample was increased (about $1 \times 10^{6}$ cells/sample), a feasible approach since about 10-20 million total cells can be isolated from a single Xenopus adult spleen. Another problem inherent to the laborious nature of the BrdU assay are the multiple washing steps necessary for the elimination of cross-reactive binding, and that pipette connected to a vacuum pump rather than decanting after centrifuging minimized cell loss.

CFSE staining of Xenopus splenocytes has been successfully used before in adoptive cell transfer experiments (23), but multiple concentrations of CFSE were tested to obtain optimal staining of splenocytes. Cytotoxicity caused by CFSE is observed at levels over $50 \mathrm{mM}$ for approximately $20 \times 10^{6}$ total cells, but a $5 \mu \mathrm{M}$ CFSE concentration was optimal and no cytotoxicity was observed (see $\mathrm{T}_{0}$ on Fig. 3 ). In contrast to thymidine incorporation assays, the CFSE proliferation assay is more informative since, with the use of flow cytometry it allows the identification of proliferating cells by specific surface markers. Furthermore, while CFSE can identify the effector cell population the serial dilutions observed in a dot plot can indicate the intensity of the effector response. In contrast, effector cell proliferation in thymidine incorporation assays may be masked by spontaneous proliferation of stimulator cells or by cell death, resulting in high background and an underestimation of the effector response. Repeated experiments have yielded consistent results and have rendered the CFSE proliferation assay as a valuable and reliable tool in our studies.

One common caveat for the use of green fluorochrome dyes in amphibian cells is autofluorescence and, given the emission spectrum of diluted CFSE, distinguishing true proliferation from autologous fluorescence using FACS can be challenging. However, this is easily circumvented by the use of an unlabeled control to determine basal level autofluorescence. Fortunately, the color intensity of the initial CFSE staining is significantly higher than autofluorescent Xenopus cells and diluting CFSE cell populations can be easily distinguished in a flow cytometry dot plot (see bottom panels in Fig. 3). Furthermore, given that autofluorescence does not vary significantly from individual to individual, comparisons between treated and untreated samples can provide a standard of autofluorescence usable in all experiments.

Overall, our studies show that both BrdU and CFSE are reliable cell labeling techniques for identifying and measuring the amount of proliferating cells in Xenopus spleen and splenocyte cultures, respectively. Both are relatively simple to perform and have the advantage of measuring cell type specific activation. It is safe to say that many methods used in mammals to assess immune cell function are easily transferable to other vertebrate

Robert et al. - In vivo and in vitro techniques for comparative study of antiviral T-cell responses in the amphibian Xenopus www.biologicalprocedures.com 
models, further bridging the gap to a complete understanding of cellular immune responses throughout vertebrate phyla.

\section{ACKNOWLEDGMENTS}

The expert animal husbandry provided by Tina Martin and David Albright is gratefully appreciated. We would also like to thank Ana Goyos and Dr. Asiya Seema Chida for critically reading the manuscript. Research support: NIH T32-AI07285 (H. M.), R24-AI-059830 (J. R.), and NSF MCB-0445509 (H. M. and J.R).

\section{REFERENCES}

1. Doherty P, Allan W, Eichelberger M. Role of ab and gd T cell subsets in viral immunity. Аппи Rev Immunol 1992; 10:123-151.

2. Du Pasquier L, Flajnik MF, Guiet C, Hsu E. Methods used to study the immune system of Xenopus (Amphibia, Anura). Immunol Methods 1985; 3:425-465.

3. Robert J, Maniero G, Cohen N, Gantress J. Xenopus as an model system to study evolution of HSP-immune system interactions. In: Methods: A Companion to Methods in Enzymology (HSP-Immune System Interactions) 2004 Ed. P. Srivastava, Academic Press. Vol 32: 42-53.

4. Carayon P, Bord A. Indentification of DNA replicating lymphocyte subsets using a new method to label the bromodeoxyuridine incorporated into the DNA. J Immunol Methods 1992; 142: 225-230.

5. Lyons AB. Analysing cell division in vivo and in vitro using flow cytometric measurements of CFSE dilution. J Immunol Methods 2000; 243:147-154.

6. Tough DF, Sprent J. Turnover of naïve and memory phenotype T cells. J Exp Med 1994; 179:1127-1135.

7. Hasbold J, Gett AV, Rush JS, Deenick E, Avery D, Jun J, Hodgkin PD. Quantitative analysis of lymphocyte differentiation and proliferation in vitro using carboxyfluorescein diacetate succinimidyl ester. Immunol Cell Biol 1999; 77:516-522.

8. Gonchoroff NJ, Katzmann JA, Surriw RM, Evans EL, Houck DW, Kline BC, Greipp PR, Loken MR. S-phase detection with an antibody to bromodeoxyuridine. Role of DNase pretreatment. J immunol Methods 1986; 93:97.

9. Vanderlaan M,Thomas CB. Characterization of monoclonal antibodies to bromodeoxyuridine. Cytometry 1985; 6: 501.

10. Lyons AB, Parish CR. Determination of lymphocyte division by flow cytometry. J. Immunol. Methods 1994; 171: 131.
11. Pantaleo G, Harari A. Functional Signatures in antiviral T cell Immunity for monitoring virus associated diseases. Nature Rev Immunol 2006; 6:417-422..

12. Klenerman P, Hill A. T cells and viral persistence: lessons from diverse infections. Nature Immunol. 2005; 6:873-879.

13. Migueles SA, Laborico AC, Shupert WL, Sabbaghian MS, Rabin R, Hallahan CW, Van Baarle D, Kostense S, Miedema F, McLaughlin M, Ehler L, Metcalf J, Liu S, Connors M. HIV specific CD8T cell proliferation is coupled to perforin expression in nonprogressors. Nature Immunol 2002; 3:1061-1068.

14. Weekes M, Wills MR, Mynard K, Hicks R, Sissons JG, Carmichael AJ. Large clonal expansions of human virusspecific memory cytotoxic $\mathrm{T}$ lymphocytes within the CD57+CD38-CD8+ T cell population. Immunology 1999; 98:443-449.

15. Estepa A, Alvarez F, Ezquerrra A, Coll JM. Viral-antigen dependence and $\mathrm{T}$ cell receptor expression in leucocytes from rhabdovirus immunized trout. Vet Immunol Immunopath 1999; 68: 73-89.

16. Gantress J, Maniero GD, Cohen N, Robert J. Development and characterization of a model system to study amphibian immune responses to iridoviruses. Virology 2003; 311(2): 254-262.

17. Robert J, Morales H, Wayne B, Cohen N, Marr S, Gantress J. Adaptive immunity and histopathology in frog virus 3infected Xenopus. Virology 2005; 332: 667-675.

18. Morales H, Robert J. In vivo characterization of primary and secondary anti- ranavirus CD8 $\mathrm{T}$ cell responses in Xenopus laevis. J Virology 2007; 81: 2240-2248.

19. Flajnik MF, Taylor E, Canel C, Grossberge, D, Du Pasquier L . Reagents specific for MHC I antigens of Xenopus. Amer Zool 1991; 31: 580-591.

20. Hsu E, Du Pasquier L. Studies in Xenopus immunoglobulins using monoclonal antibodies. Mol Immunol 1984; 21:257-270.

21. Cohen N. Phylogeny of Lymphocyte Structure and Function. Amer Zool 1975; 15(1): 119-133.

22. Maniero GD, Morales H, Gantress J, Robert J. Generation of a long-lasting, protective, and neutralizing antibody response to the ranavirus FV3 by the frog Xenopus. Devel Comp Immunol 2006; 30: 649-657.

23. Maniero GD, Robert J. Phylogenetic conservation of gp96mediated antigen specific cellular immunity: New evidence from adoptive cell transfer in Xenopus. Transplantation 2004; 78(10): 1415-1421.

Robert et al. - In vivo and in vitro techniques for comparative study of antiviral T-cell responses in the amphibian Xenopus www. biologicalprocedures.com 


\section{PROTOCOLS}

Protocol I: In vivo BrdU incorporation assay

Reagents:

- Amphibian grade PBS- PBS $+30 \%$ distilled $\mathrm{H}_{2} \mathrm{O}$

- 5g/L Tricaine methane sulfonate (TMS222; Crescent Research Chemical, Phoenix AZ, cat. \# 886-86-2) buffered with sodium bicarbonate $0.5 \mathrm{~g} / \mathrm{L}$.

- Staining Buffer 1: APBS containing $1 \%$ BSA and $0.01 \% \mathrm{NaN}_{3}$

- DNAse buffer: $40 \mathrm{mM}$ Tris $\mathrm{HCl}$, pH8.0 with $10 \mathrm{mM} \mathrm{NaCl}$ and $6 \mathrm{mM} \mathrm{MgCl}_{2}$

- Permeabilization buffer: APBS containing 1\% Paraformaldehyde (Sigma) and 0.05\% Tween 20 (Sigma)

- Staining Buffer 2: APBS containing $0.01 \%$ Tween 20

FV3 infection and Incubation with BrdU:

In vivo BrdU incubation works best with outbred frogs of about 2 years of age or 2-3 inches long. Outbred frogs are injected intraperitoneally with $1-5 \times 10^{6}$ pfus of FV3 in sterile APBS. The animals are kept in our facility away from uninfected animals for 4 days. Each frog is placed separately in a $500 \mathrm{ml}$ beaker with $100 \mathrm{ml}$ dechlorinated water containing $1 \mathrm{mg} / \mathrm{ml}$ BrdU 2 days before sacrifice.

\section{Splenocyte harvest:}

1. At time of harvest, euthanize the animals in 5g/L TMS222.

2. Remove the spleen and keep in cold APBS on ice.

3. Scrape the spleen (sterile technique not necessary) with a nylon mesh and wash the cells 2 times with APBS and 1 time with Staining Buffer 1.

4. Count the cells and distribute them at $500,000-1 \times 10^{6}$ cells per sample

\section{Cell staining:}

1. After washing, resuspend the cells in $100 \mathrm{ml} \mathrm{mAb}$ diluted at $1 \mathrm{mg} / \mathrm{ml}$ (final concentration) with Staining buffer 1, incubate for $30 \mathrm{~min}-1 \mathrm{hr}$ on ice.

2. Wash 2 times with Staining Buffer 1 .

3. Resuspend cells in $100 \mathrm{ml}$ secondary $\mathrm{Ab}$ (if needed) diluted in staining buffer 1, and incubate in the dark on ice for 30 min. Keep cells in the dark from now on.

4. Wash 2 times with staining buffer 1 .

5. Resuspend in 50-100 $\mathrm{\mu l}$ of permeabilization buffer at room temperature for $30 \mathrm{~min}$ in the dark.

6. Wash 2 times with DNase buffer.

7. Resuspend cells in $100 \mathrm{ml}$ DNase buffer $+3 \mathrm{ml}$ DNase I at $37^{\circ} \mathrm{C}$ for $30 \mathrm{~min}$.

8. Wash 2 times in staining buffer 2 .

9. Incubate in $3 \mathrm{ml}$ anti-BrdU-FITC in $25 \mathrm{ml}$ of staining buffer 2 on ice for $30 \mathrm{~min}-1 \mathrm{hr}$.

10. Wash 2 times with Staining buffer 2 and resuspend in at least $100 \mathrm{ml}$ staining buffer.

11. Analyze by FACS.

Protocol II: CFSE proliferation assay

Reagents:

- CFSE: Dissolve CFSE powder in DMSO to a $5 \mathrm{mM}$ stock. Aliquot $100 \mathrm{ml}$ in eppendorf tube. Keep in $-20^{\circ} \mathrm{C}$ in a dark and dry area.

Robert et al. - In vivo and in vitro techniques for comparative study of antiviral T-cell responses in the amphibian Xenopus www.biologicalprocedures.com 
- ASF-A6-MSF: Iscove DMEM basal medium diluted at the amphibian osmolarity and containing 5\% FBS, $10 \mathrm{mg} / \mathrm{ml}$ of Kanamycin and 20\% supernatant from the A6 kidney fibroblast cell line (ATCC: CCL 102; see ref. 17 for more details).

- Staining Buffer 1: APBS with $1 \%$ BSA and $0.01 \% \mathrm{NaN}_{3}$.

\section{Priming:}

Outbred frogs are primed by intraperitoneal injection of $1-5 \times 10^{6}$ pfus FV3 in sterile APBS. The animals are kept in our facility away from uninfected animals for 3-4 weeks.

\section{Peritoneal leukocyte isolation and in vitro infection:}

Peritoneal leukocytes (PLs) from infected and uninfected animals are isolated by peritoneal lavage as described in detailed elsewhere (2). Anesthetized frogs (a $0.1 \%$ aqueous solution buffered with sodium bicarbonate of TMS222) are injected intraperitoneally (sterile syringe with a 22 gauge 11/2 inch needle) with 5 to $10 \mathrm{ml}$ (depending of the size of animal) of APBS prewarmed at room temperature. The frog is then gently massaged then the solution injected is recovered into a sterile tube by puncture with a $22 \mathrm{G} 1 \frac{1}{2}$ gauge needle. Cells are washed twice with cold APBS, counted with a hemacytometer and seeded in a 24well plate at 100,000-500,000 cells per well in 1ml ASF-A6 medium. Cells are then infected with FV3 at 1 MOI (or $100,000-500,000$ pfu per well), and incubated overnight at $27^{\circ} \mathrm{C}$ in a $\mathrm{CO}_{2}$ chamber.

\section{Day 1 Co-culture Splenocytes:}

1. Euthanize frogs in $5 \mathrm{~g} / \mathrm{L}$ TMS222 and isolate the spleen using sterile technique.

2. Scrape the spleen and wash with cold sterile APBS. Ensure proper suspension.

3. Incubate with $520 \mathrm{mM}^{*} \mathrm{CFSE}$ at $50 \times 10^{6}$ cells $/ \mathrm{ml}$ at $27^{\circ} \mathrm{C}$ in the dark for $20 \mathrm{~min}$.

*Ex vivo splenocytes stain well at $5 \mu \mathrm{M}$ concentration, however, a CFSE titration should be done to determine best labeling concentration for cells of interest.

4. Wash cells well 2-3x with cold APBS.

5. Distribute splenocytes with corresponding PLs at a one to one ratio (different stimulator to primed splenocyte ratios may also be done).

6. Incubate the plates at $27^{\circ} \mathrm{C}$ in a $\mathrm{CO}_{2}$ chamber for $48-96$ hours.

7. Total co-culture is used for cell surface labeling.

\section{Day 2-Day 6 Cell surface labeling:}

1. Resuspend cells and wash 2 times in cold APBS and 1 time in cold staining buffer 1 .

2. Distribute to at least 100,000 cells per stain.

3. Add $100 \mathrm{ml}$ of diluted primary antibody and incubate on ice for $30 \mathrm{~min}-1 \mathrm{hr}$.

4. Wash cells 2 times in staining buffer 1 .

5. Add $100 \mathrm{ml}$ of diluted secondary antibody and incubate on ice $30 \mathrm{~min}-1 \mathrm{hr}$.

6. Wash cells 2 times in staining buffer and resuspend in at least $100 \mu \mathrm{l}$ staining buffer.

7. Analyze by FACS. Collect at least 20,000 total events. 